\title{
Acidentes ocupacionais com material biológico em Odontologia: uma responsabilidade no ensino
}

\author{
Seani Neumann de Paiva*; Wanessa Christine de Souza Zaroni**; Mariana Ferreira Leite ${ }^{* * ;}$ Patricia \\ Rocon Bianchi ***; Teresa Cristina Rangel Pereira*****
}

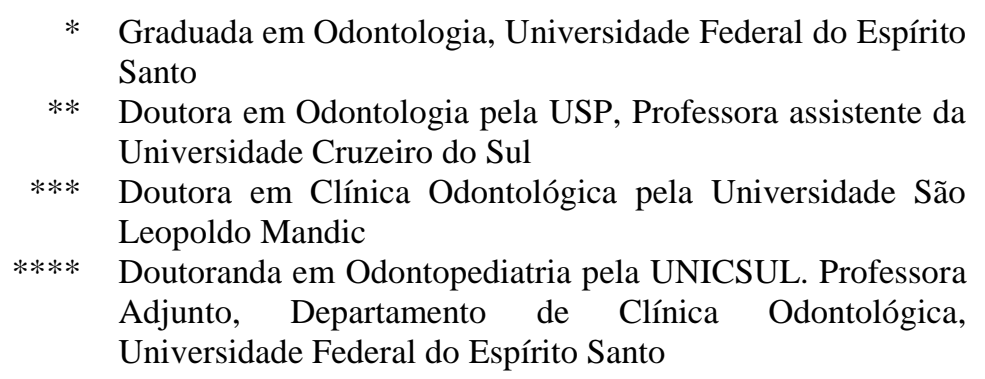

Recebido em 04/02/2017. Aprovado em 17/07/2017.

\begin{abstract}
RESUMO
Durante a graduação em Odontologia o aluno enfrenta situações que podem causar danos à própria saúde, visto que estão expostos a diversos riscos ocupacionais com material biológico. Com o intuito de contribuir com dados que auxiliem na formação acadêmica, propõe-se verificar a ocorrência, as características dos acidentes com material biológico, o conhecimento e a aplicação de ações preventivas e condutas pós-exposição. Trata-se de uma pesquisa observacional, transversal e descritiva utilizando um questionário de autopreenchimento aplicado a 195 estudantes do curso de Odontologia que tenham contato com procedimentos clínicos. Dos entrevistados, 23,6\% sofreram acidentes ocupacionais, com predomínio do sexo feminino $(78,3 \%)$, frequentemente acometendo as mãos $(66,7 \%)$, e a maioria causados por sonda exploradora (30,1\%). Observou-se que 34,9\% sofreram acidentes percutâneos, somente $18,7 \%$ procurou auxílio médico e $6,3 \%$ relataram não usar equipamentos de proteção individual (EPI) no momento do acidente. Lavar o local afetado com água e sabão $(54,7 \%)$ foi a principal conduta pós-exposição. A ocorrência de acidentes frente ao contato com material contaminado desperta atenção devido ao uso incompleto ou não uso de EPI, além da pouca procura por atendimento médico após os acidentes. Os acadêmicos relataram ter conhecimento da legislação relacionada à saúde do trabalhador e de como proceder em caso de acidentes ocupacionais. É necessário o desenvolvimento de estratégias de ensino que incluam uma supervisão mais eficaz, medidas que motivem a atenção para redução dos acidentes ocupacionais e o aumento da notificação de casos, de maneira contínua.
\end{abstract}

Descritores: Exposição a Agentes Biológicos. Riscos Ocupacionais. Prevenção de Acidentes. Estudantes de Odontologia. Notificação de Acidentes de Trabalho. 


\section{INTRODUÇÃO}

A maioria dos procedimentos realizados na Odontologia implica em contato com o paciente, favorecendo o risco de acidentes laborais com exposição a agentes biológicos, pois utiliza instrumentos perfurocortantes e equipamentos que produzem aerossóis potencialmente infectantes ${ }^{1}$. Diversos estudos mostram que os acadêmicos de Odontologia são um grupo frequentemente acometido por acidentes ocupacionais $^{1-7}$.

A ocorrência de acidentes com material biológico possibilita a transmissão de patógenos via secreções e fluidos corpóreos. Entre eles destacam-se o vírus da Hepatite B (HBV), o vírus da Hepatite C (HCV) e o vírus da Imunodeficiência Humana (HIV) ${ }^{8}$. De acordo com o Ministério da Saúde no ano de 2015 foram registrados 51 casos de Hepatite B e 86 casos de Hepatite C contraídas por acidentes ocupacionais ${ }^{9}$. Estudo recente, também com estudantes de Odontologia, encontrou alta prevalência de acidentes ocupacionais, com mais da metade deles não tendo sido informados aos surpevisores $^{6}$. O uso correto de equipamentos de proteção individual (EPIs) reduz os riscos de exposição a materiais biológicos, contudo muitos estudantes não os utilizam ${ }^{10-12}$.

Todos os casos de acidente com material biológico devem ser comunicados ao Instituto Nacional de Seguridade Social (INSS) por meio da Comunicação de Acidente de Trabalho (CAT) e ao Ministério da Saúde por meio do Sistema de Informação de Agravos de Notificação (SINAN), conforme previsto na Portaria n. ${ }^{\circ} 777$, de 28 de abril de 2004, do Ministério da Saúde ${ }^{13}$, reforçada pela portaria $n^{\circ} 104$ de 25 de janeiro de $2011^{14}$. Além disso, a instituição deve manter um registro interno com todos os dados do acidente ${ }^{15}$. Porém, na maioria das universidades, a notificação é deficiente $e^{6,10,16-19}$.

As instituições de ensino de Odontologia devem preparar os acadêmicos a racionalizar e otimizar a aplicação de conhecimentos e metodologias de biossegurança que melhorem as ações e decisões da futura prática profissional ${ }^{20}$. A exposição a material biológico no ambiente acadêmico precisa ser vista com grande atenção durante a formação dos alunos. O capítulo III das contribuições ao aprimoramento das Diretrizes Curriculares Nacionais (DCN) do Curso de Graduação em Odontologia em seu art. 13º, descreve que a formação do cirurgião-dentista tem por objetivo dotar o profissional dos conhecimentos requeridos para o exercício de competências específicas, como pode ser observado no inciso XIX: "Promover o autocuidado para prevenção de acidentes de trabalho e doenças ocupacionais" ${ }^{21}$.

Para contribuir com dados que auxiliem na formação do estudante de Odontologia e possibilitem ações preventivas, o objetivo deste trabalho foi verificar a ocorrência, as características dos acidentes ocupacionais, o conhecimento e a aplicação de ações preventivas dos acadêmicos do Curso de Odontologia de uma Universidade Federal da região Sudeste do Brasil.

\section{MÉTODOS}

Trata-se de um estudo observacional, transversal, descritivo com 195 acadêmicos do Curso de Odontologia de uma Universidade Federal, que cursavam entre o $2^{\circ}$ e $9^{\circ}$ períodos no ano de 2012, distribuídos em 2 grupos: $2^{\circ}$ ao $5^{\circ}$ período $n=100(51,3 \%)$ e $6^{\circ}$ ao $9^{\circ}$ período $n=95$ $(48,7 \%)$. Os critérios de inclusão para a amostra foram exercer atividades clínicas e autorizar a coleta de dados mediante assinatura do Termo de Consentimento Livre e Esclarecido.

Foi utilizado um questionário autoaplicável, composto de questões objetivas e subjetivas, com dados do indivíduo (sexo e idade) e da ocorrência do acidente ocupacional 
(período do curso, disciplina cursada no momento do acidente, procedimento $\mathrm{e}$ instrumento causador, região corpórea afetada, utilização de equipamentos de proteção individual, gravidade, condutas após o acidente e conhecimentos sobre sua prevenção). Nos casos em que o participante havia sofrido mais de uma injúria, ficha acessória foi entregue para que fosse preenchida com as características de cada acidente, separadamente.

Os resultados foram apresentados com números absolutos e proporcionais para descrição dos temas investigados. Os dados foram armazenados em planilha Excel $^{\circledR}$ e analisados pelo software SPSS $^{\circledR}$ versão 20.0 (software Statistical Package for the Social Science, 20.0 - SPSS Inc. Chicago, IL, EUA), utilizando o teste qui-quadrado para comparação de proporções. $\mathrm{O} p$-valor $<0,05$ foi considerado estatisticamente significante.

Este estudo foi aprovado pelo Comitê de Ética com o parecer 95.728 (CAAE: 03015012.7.0000.5060) e não recebeu financiamento para sua realização.

\section{RESULTADOS}

A maioria dos entrevistados (147 indivíduos - 75,4\%) era do sexo feminino, com o predomínio da faixa etária de 20 a 30 anos $(83,6 \%)$.

Foram relatados 63 acidentes, com 46 pessoas atingidas (23,6\% do total da amostra). Houve um predomínio do sexo feminino $(78,3 \%)$ em relação ao número de ocorrência total de acidentes, entretanto, os acadêmicos que sofreram mais de dois acidentes eram do sexo masculino $(4,3 \%)$. As regiões mais afetadas, os instrumentais envolvidos e a distribuição dos acidentes quanto ao momento de sua ocorrência são apresentados na tabela 1 .

A ocorrência de acidentes ocupacionais e o conhecimento sobre a legislação relacionada à saúde do trabalhador são observados na tabela 2 . No grupo dos acadêmicos do $2^{\circ}$ ao $5^{\circ}$ período, $8 \%$ sofreram acidentes, sendo que $25 \%$ destes, mais de uma vez. No grupo dos acadêmicos do $6^{\circ}$ ao $9^{\circ}$ período, foi observado que $40,0 \%$ acidentaram-se, sendo 34,2\% destes mais de uma vez. $\mathrm{O}$ índice de acidentes por acadêmico foi de 0,32 .

A maioria dos acadêmicos afirmou ter recebido instruções de quais medidas seguir em caso de acidente $(81,5 \%)$ e de como evitá-lo $(93,3 \%)$ (tabela 2).

Os alunos mais afetados cursavam o $6^{\circ}$ e $5^{\circ}$ período $(28,6 \%$ e $22,2 \%$ dos acidentes respectivamente). $\mathrm{O}$ maior número de acidentes ocorreu nas disciplinas de Odontologia Restauradora Clínica (9,5\%), Clínica Integrada de Atenção Primária (4,8\%), Endodontia (4,8\%) e Prótese Clínica (4,8\%). Em quatro casos $(6,3 \%)$ os estudantes relataram não lembrar onde tal incidente ocorreu e em três casos $(4,8 \%)$ foi em campos de estágio extramuros.

Quanto ao tipo de acidente ocorrido, observou-se que os respingos em pele íntegra $(49,2 \%)$ e os ferimentos percutâneos $(34,9 \%)$ foram os mais comuns, embora $14,3 \%$ tenham sofrido respingo em mucosa e 1,6\% respingo em pele não íntegra. Dos participantes que sofreram as injúrias mais graves, como a exposição do material biológico em pele não íntegra, exposição em mucosa e perfuração, observados em 32 casos (50,8\% do total dos acidentes), somente $18,7 \%$ procuraram ajuda médica.

Em relação ao uso do EPI no momento do acidente, verificou-se que 4 (6,3\%) acadêmicos relataram não usar qualquer equipamento, 33 $(52,4 \%)$ utilizaram de forma incompleta e 26 $(41,3 \%)$ estavam com equipamentos adequados para proteção preconizada para a tarefa que estava sendo realizada (gráfico 2).

As condutas pós-exposição adotadas pelos acidentados são apresentadas na tabela 3. Os atos 
mais comuns foram lavar o local com água e sabão e utilizar agente antisséptico. Quando consideradas as condutas preconizadas pelo Ministério da Saúde de acordo com o Protocolo de Exposição a Acidentes Ocupacionais específicos para cada tipo de exposição ${ }^{8}, 63,5 \%$ das ações pós-acidentes foram corretamente realizadas. Houve diferença significativa entre os grupos para a ação de passar antisséptico na ferida.

Foi verificada correlação significativa entre acadêmicos dos períodos iniciais/finais e os acidentes ocupacionais $(\mathrm{p}=0,00016)$, com um aumento de acidentados nos períodos finais. Não foi observada correlação $(p=0,6336)$ entre o número de acidentes e o recebimento de informações sobre como evitá-los (tabela 3).

Tabela 1. Prevalência dos acidentes com material biológico segundo a região atingida, o instrumento que o ocasionou e o momento de sua ocorrência

\begin{tabular}{|c|c|c|}
\hline \multirow{2}{*}{ Variáveis } & \multicolumn{2}{|c|}{ Alunos } \\
\hline & $\mathbf{n}$ & $\%$ \\
\hline \multicolumn{3}{|l|}{$\overline{\text { Região do corpo atingida }}$} \\
\hline Mãos & 42 & $66,7 \%$ \\
\hline Braços & 2 & $3,2 \%$ \\
\hline Olhos & 10 & $15,9 \%$ \\
\hline Face & 8 & $12,6 \%$ \\
\hline Outro & 1 & $1,6 \%$ \\
\hline \multicolumn{3}{|l|}{ Instrumento causador } \\
\hline Agulha de anestesia & 5 & $7,9 \%$ \\
\hline Sonda exploradora & 19 & $30,1 \%$ \\
\hline Cureta periodontal & 3 & $4,8 \%$ \\
\hline Agulha de seringa & 1 & $1,6 \%$ \\
\hline Lâmina de bisturi & 1 & $1,6 \%$ \\
\hline Sindesmótomo & 1 & $1,6 \%$ \\
\hline Agulha do fio de sutura & 3 & $4,8 \%$ \\
\hline Lima endodôntica & 5 & $7,9 \%$ \\
\hline Broca & 2 & $3,2 \%$ \\
\hline Seringa tríplice & 10 & $15,9 \%$ \\
\hline Escova Robson para profilaxia & 5 & $7,9 \%$ \\
\hline Não sei & 3 & $4,8 \%$ \\
\hline Outros & 5 & $7,9 \%$ \\
\hline \multicolumn{3}{|l|}{ Momento do acidente } \\
\hline Enquanto atendia o paciente & 35 & $55,6 \%$ \\
\hline Carregando os instrumentais & 8 & $12,7 \%$ \\
\hline Lavando os instrumentais & 14 & $22,2 \%$ \\
\hline Embalando instrumentais para esterilização & 6 & $9,5 \%$ \\
\hline Total & 63 & $100 \%$ \\
\hline
\end{tabular}


Tabela 2. Ocorrência de acidentes ocupacionais entre acadêmicos, conhecimento sobre a legislação e instruções sobre acidentes de trabalho em Odontologia

\begin{tabular}{|c|c|c|c|c|c|c|}
\hline \multirow{3}{*}{ Variáveis } & \multicolumn{6}{|c|}{ Grupo } \\
\hline & \multicolumn{2}{|c|}{$\begin{array}{c}\text { Acadêmicos } \\
\left(2^{\circ} \text { ao } 5^{\circ} \text { período }\right)\end{array}$} & \multicolumn{2}{|c|}{$\begin{array}{c}\text { Acadêmicos } \\
\left(6^{\circ} \text { ao } 9^{\circ} \text { período }\right)\end{array}$} & \multicolumn{2}{|c|}{ Total } \\
\hline & $\mathbf{n}$ & $\%$ & $\mathbf{n}$ & $\%$ & $\mathbf{n}$ & $\%$ \\
\hline \multicolumn{7}{|l|}{ Sofreu acidente* } \\
\hline Sim & 8 & $8,0 \%$ & 38 & $40,0 \%$ & 46 & $23,6 \%$ \\
\hline Não & 92 & $92,0 \%$ & 58 & $60,0 \%$ & 149 & $76,4 \%$ \\
\hline \multicolumn{7}{|l|}{ Mais de uma vez } \\
\hline Sim & 2 & $25,0 \%$ & 13 & $34,2 \%$ & 15 & $32,6 \%$ \\
\hline Não & 6 & $75,0 \%$ & 25 & $65,8 \%$ & 31 & $67,4 \%$ \\
\hline Total & 8 & $100,0 \%$ & 38 & $100,0 \%$ & 46 & $100,0 \%$ \\
\hline \multicolumn{7}{|c|}{ Conhece a NR no 32} \\
\hline Sim & 68 & $68,0 \%$ & 42 & $44,2 \%$ & 110 & $56,4 \%$ \\
\hline Não & 32 & $32,0 \%$ & 53 & $55,8 \%$ & 85 & $43,6 \%$ \\
\hline \multicolumn{7}{|c|}{ Recebeu instrução do que fazer após acidente de trabalho } \\
\hline Sim & 81 & $81,0 \%$ & 78 & $82,1 \%$ & 159 & $81,5 \%$ \\
\hline Não & 19 & $19,0 \%$ & 17 & $17,9 \%$ & 36 & $18,5 \%$ \\
\hline \multicolumn{7}{|c|}{ Recebeu instrução de como evitar um acidente de trabalho** } \\
\hline Sim & 93 & $93,0 \%$ & 89 & $93,7 \%$ & 182 & $93,3 \%$ \\
\hline Não & 7 & $7,0 \%$ & 6 & $6,3 \%$ & 13 & $6,7 \%$ \\
\hline Total & 100 & $100,0 \%$ & 95 & $100,0 \%$ & 195 & $100,0 \%$ \\
\hline
\end{tabular}

*Diferença estatística significativa entre acadêmicos dos períodos iniciais/finais e acidentes, $\mathrm{p}=0,00016$

** Não foi estatisticamente significativo, $\mathrm{p}=0,6336$

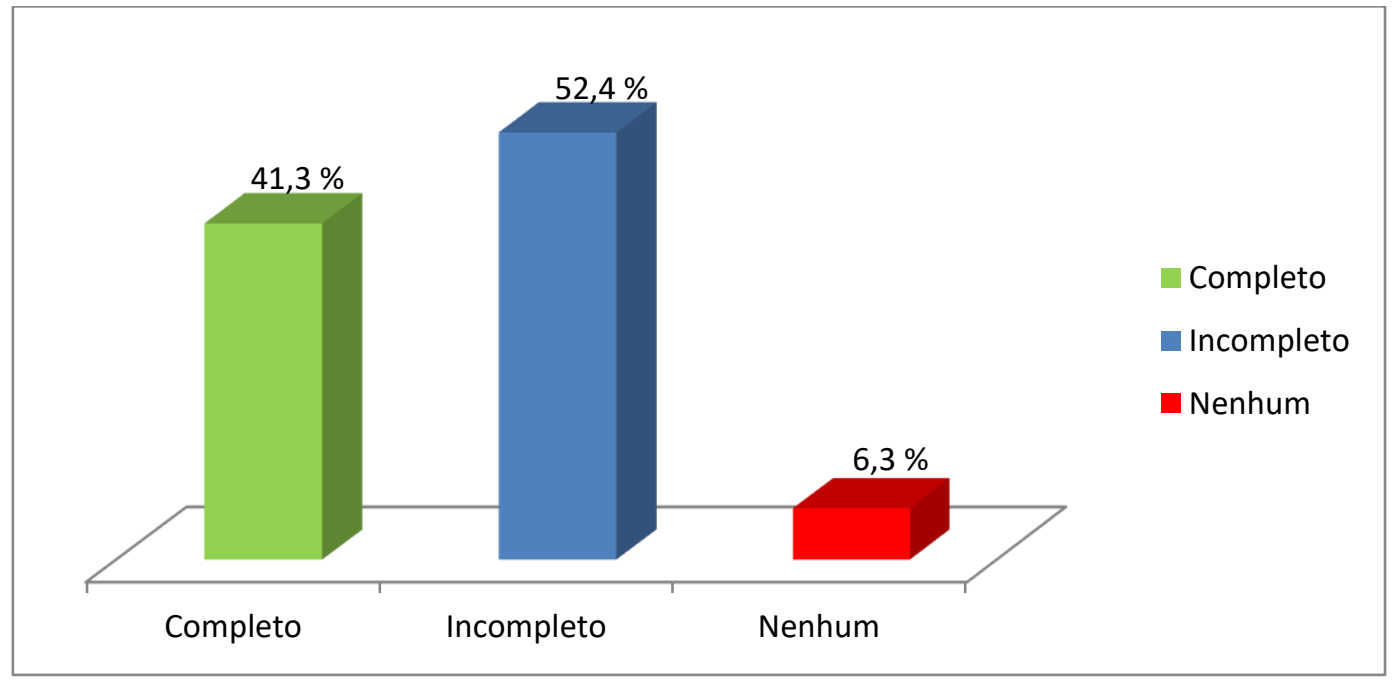

Gráfico 1. Uso de equipamento de proteção individual no momento do acidente 
Tabela 3. Condutas pós-exposição adotadas pelos acidentados

\begin{tabular}{|c|c|c|c|c|c|}
\hline \multirow[t]{2}{*}{ Condutas } & \multicolumn{2}{|c|}{$\begin{array}{c}\text { Acadêmicos } \\
\left(2^{\circ} \text { ao } 5^{\circ} \text { período }\right)\end{array}$} & \multicolumn{2}{|c|}{$\begin{array}{c}\text { Acadêmicos } \\
\left(6^{\circ} \text { ao } 9^{\circ} \text { período }\right)\end{array}$} & \multirow[t]{2}{*}{$p$-valor } \\
\hline & $\mathbf{n}$ & $\%$ & $\mathbf{n}$ & $\%$ & \\
\hline Nenhuma & 2 & 20,0 & 13 & 24,5 & 0,922 \\
\hline Interrompeu o procedimento & 3 & 30,0 & 13 & 24,5 & 0,976 \\
\hline Lavou com água e sabão & 5 & 50,0 & 29 & 54,7 & 0,942 \\
\hline Passou antisséptico & 7 & 70,0 & 11 & 20,8 & $0,005^{*}$ \\
\hline Limpou com $\mathrm{H}_{2} \mathrm{O}_{2}$ & 0 & 0,0 & 5 & 9,4 & 0,710 \\
\hline Fez um curativo & 2 & 20,0 & 1 & 1,9 & 0,098 \\
\hline \multicolumn{6}{|c|}{ De acordo com protocolo do Ministério da Saúde } \\
\hline Correta & 5 & 50,0 & 35 & 66,0 & 0,545 \\
\hline Incorreta & 5 & 50,0 & 18 & 34,0 & 0,545 \\
\hline Total & 10 & $100,0 \%$ & 53 & $100,0 \%$ & - \\
\hline
\end{tabular}

*Estatisticamente significativo

\section{DISCUSSÃO}

A educação em Biossegurança e controle de infecção é imprescindível e tem que ser de constante aprendizado. $\mathrm{O}$ cumprimento dos protocolos entre os acadêmicos deve ser incentivado e cobrado nos ambientes de ensino Odontológico ${ }^{5,6,20}$. Os acidentes com materiais biológicos nos cursos de graduação em Odontologia devem ser minimizados, uma vez que podem resultar em doenças e agravos à saúde do estudante e futuro profissional, tanto do ponto de vista físico quanto do aspecto psicológico $^{22,23}$.

Os estudantes da área de saúde estão em terceiro lugar entre as ocupações que sofrem mais acidentes ${ }^{2}$ e os acadêmicos de Odontologia são os graduandos da área de saúde com o maior índice de acidentes com material biológico e perfurocortante quando comparados aos graduandos em Enfermagem, Farmácia e Medicina ${ }^{4}$. Como as instituições são responsáveis pela constante avaliação do processo ensinoaprendizagem $^{20}$, e esses dados têm se repetido em outros estudos, a temática deveria motivar debate sobre a atenção à saúde dos próprios estudantes nos meios acadêmicos, não somente no cuidado com o aprendizado técnico, mas também no favorecimento de condições de segurança e bem-estar.

A taxa de acadêmicos expostos a material biológico relatada neste estudo foi de $23,6 \%$, valor semelhante ao encontrado por Orestes-Cardoso et al. (2009) ${ }^{22}$, que obtiveram uma taxa de acidentes de $25,3 \%$ em acadêmicos de Odontologia. Neste estudo, o maior índice de acidentes ocorreu com os acadêmicos de períodos mais avançados (40\%), em relação aos alunos dos períodos iniciais (8\%). Esses dados estão de acordo com a literatura e podem ser justificados devido ao maior acúmulo de horas clínicas ${ }^{5,9,16}$.

O sexo feminino é comumente o mais acometido nesse tipo de acidente $e^{3,22,24-30}$. Tal resultado pode ser atribuído ao maior número de mulheres na área da saúde ${ }^{28}$. Essa interpretação pode ser aplicada no presente 
estudo, uma vez a amostra foi composta por um número consideravelmente maior de indivíduos do sexo feminino $(75,4 \%)$. Acredita-se também que, devido ao fato de as mulheres geralmente serem mais cuidadosas com a saúde, elas se recordem e notifiquem mais os acidentes.

A região mais afetada foi a das mãos (66,7\%), concordando com outros estudos $^{3,10,25,28}$. Os olhos $(15,9 \%)$ e a face $(12,7 \%)$ também foram atingidos, o que pode estar relacionado à utilização incorreta ou não utilização do EPI. Seria importante, e correto de acordo com as DCN, que os discentes fossem estimulados por alguma estratégia de ensino a conhecerem e aplicar as normas dos trabalhadores da área da saúde bucal $^{20}$.

Os instrumentos causadores de acidentes frequentemente citados na literatura são as sondas exploradoras ${ }^{3,18,25,31}$ e as agulhas para anestesia ${ }^{3,10,18,25,31}$, semelhantes aos dados encontrados nessa pesquisa. No presente estudo a sondas exploradoras apresentaram a maior frequência de acidentes $(30,1 \%)$. Embora as agulhas sejam citadas como os instrumentos com maiores índices de acidentes, nesta pesquisa o valor foi mais baixo $(7,9 \%)$, devido ao grande reforço por parte dos docentes para que os alunos não reencapem as agulhas, posicionem a seringa carpule em local separado dos demais instrumentos e com a agulha voltada para fora do campo operatório. A escova Robson, conquanto pareça um material "inofensivo", considerado com baixo risco de transmissão biológica e de pouco potencial danoso, apresentou os mesmos índices de acidentes $(7,9 \%)$ que as agulhas de anestesia e limas endodônticas, despertando a necessidade de atenção para seu correto manuseio.
A seringa tríplice provocou a segunda maior frequência de exposição do aluno a material biológico, pode ter contribuído pelo alto índice de acidentes em pele e mucosa (principalmente os olhos) e estavam associados ao uso incorreto do EPI. Embora pareçam apresentar pouco potencial danoso não podemos desconsiderar o risco de contaminação, principalmente se houver contato com pele não íntegra e mucosas.

Um dado interessante observado no estudo foi que as disciplinas que realizam os procedimentos semicríticos (em que há contato com secreções orgânicas sem invadir o sistema vascular) registraram o maior número de acidentes. $\mathrm{Na}$ disciplina de Odontologia Restauradora Clínica ocorreu o maior índice de exposição a material biológico, seguido da Clínica Integrada de Atenção Primária, Endodontia e Prótese Clínica. Considera-se que isso se deva ao fato de os estudantes estarem menos atentos aos possíveis acidentes ou acreditarem que os procedimentos realizados nessas clínicas sejam de baixo risco de contaminação. Lima et al. $(2016)^{18}$ observaram que as disciplinas de Clínica Integrada e Clínica Odontológica apresentaram os maiores índices de acidentes perfurocortantes e sugerem que isso foi devido ao aumento de atividades do futuro profissional. Essas informações justificam a necessidade de a instituição de ensino se sensibilizar e motivar ações relacionadas à biossegurança, uma vez que faz parte do seu escopo a promoção do autocuidado para prevenção de acidentes de trabalho e doenças ocupacionais ${ }^{20,21}$.

Momentos de ansiedade devido a atividades com pacientes ${ }^{25}$, pressa, cansaço, nervosismo, estresse, volume de trabalho, falta de atenção às medidas de precaução e ao uso de EPIs, além da falha pessoal, são as 
causas da exposição à material biológico ou com materiais perfurocortantes na Odontologia $^{32}$. A maioria dos acadêmicos deste estudo acidentou-se durante o atendimento, estando de acordo com a literatura ${ }^{3,10,18,22,23,28}$. Entretanto, não se pode negligenciar os momentos pós atendimento, uma vez que os acidentes também ocorreram durante o transporte e manipulação dos instrumentos, como lavagem e preparo do material para esterilização.

Embora a maioria dos estudantes tenha relatado utilizar EPI no momento do acidente, $52,4 \%$ o usavam de maneira incompleta e $6,4 \%$ não utilizavam qualquer EPI, o que demonstra uma atitude de total descompromisso com a própria saúde. A percepção de que o procedimento clínico não é de risco, a pressa na realização do atendimento, o desconforto ou dificuldade de adaptação foram os possíveis fatores para o uso incorreto dos EPI. Acrescentamos ainda o fato de que, em alguns momentos, a ausência de uma cobrança eficaz do professor ou responsável com a paramentação correta dos alunos possa favorecer tal situação. Medidas de reforço sobre os EPI devem ser adotadas para que os alunos não negligenciem seu correto uso, visto que os hábitos adquiridos na graduação são levados para a vida profissional ${ }^{9}$.

Resultados encontrados por Miotto e Rocha (2012) ${ }^{11}$, em um estudo realizado na mesma instituição, verificaram que $23,8 \%$ dos alunos não usavam EPI. Comparando com os resultados deste trabalho, verificamos que houve redução do número de indivíduos que não usavam proteção no momento do acidente. Tal situação permitenos inferir que houve melhora nas ações relatadas pelos acadêmicos quanto ao seu uso, pois nesse intervalo ocorreu a criação da disciplina específica de Biossegurança na instituição.

Considera-se exposição de alto risco quando esta ocorre devido a ferimento por agulha ou outros objetos cortantes, contato em pele lesada e respingo em mucosa, olhos ou dentro da boca ${ }^{33}$. Analisando a gravidade da exposição ao material biológico, os mais observados foram respingos e acidentes percutâneos, corroborando com outros estudos $3,12,16,17,28,34$. Condutas pós-exposição incluem procedimentos que devem ser realizados após a exposição a sangue e fluidos corpóreos do paciente para prevenir a provável transmissão de microrganismos, incluindo lavagem imediata da área exposta, determinação do risco associado à exposição, avaliação do paciente fonte em relação a HBV, HVC e HIV, vacinação contra hepatite, utilização de drogas antiretrovirais e avaliação e controle do profissional da área da saúde ${ }^{1,21}$. Entre as condutas imediatas após o acidente, a mais relatada foi lavar o local atingido com água e sabão $0^{5,12,16,18,25,29,30,34}$.

O Ministério da Saúde não indica passar soluções antissépticas sobre o local afetado, porém também não as contraindica ${ }^{8}$. Shaghaghian et al. (2014) ${ }^{1}$ observaram que $77 \%$ dos cirurgiões-dentistas usaram desinfetantes tópicos em ferimentos com instrumentos perfurocortantes. Esse procedimento foi realizado por $28,6 \%$ dos acidentados nesta pesquisa. Foi verificado que, em relação a essa atitude, houve diferença significativa entre os grupos formados pelos alunos dos períodos iniciais do curso em relação aos períodos finais do curso. Pinelli et al. $(2016)^{6}$ também observaram que houve aumento de exposições acidentais ao longo da carreira 
acadêmica, com o maior número de acidentados nos períodos finais. Acrescentaram, ainda, que a maior experiência clínica não significa maiores cuidados com a prevenção, observaram a redução ao medo de se ferir à medida que os acadêmicos vão ganhando experiência ${ }^{35}$.

Mesmo sabendo da importância de se interromper o atendimento e de identificar a área acometida imediatamente, somente por $34,8 \%$ dos acidentados desta pesquisa realizaram tal procedimento. Um estudo de Garcia e Blank (2008) ${ }^{17} \mathrm{com}$ cirurgiõesdentistas mostrou que $26,7 \%$ interromperam o atendimento que estavam realizando, porém melhores que o de Shaghaghian et al. $(2014)^{1}$ em que somente $5 \%$ verificaram a área afetada.

A procura por atendimento médico após exposição a materiais biológicos é importante e deve ser feita o mais rápido possível, sobretudo nos casos de acidentes percutâneos e respingos em pele não íntegra ou mucosa. No presente estudo, apenas $18,7 \%$ dos acidentados que sofreram injúrias mais graves procuraram assistência médica. Estudos demonstram que grande parte dos estudantes de Odontologia não relatam a ocorrência da exposição aos seus instrutores ou a subnotificam ${ }^{6,35}$. Entre as justificativas mais comuns para não procurarem o auxílio médico, estão a crença de que a exposição ocorrida seja de baixa severidade, não ser clinicamente significante e estarem muito ocupados com as suas atividades ${ }^{29,34}$. Devese enfatizar que é nessa assistência médica que, uma vez configurado o acidente com material biológico, será realizada comunicação ao INSS por meio da Comunicação de Acidente de Trabalho (CAT) e ao Ministério da Saúde. Portanto, a subnotificação das exposições percutâneas e mucocutâneas é comum e permanece problemática $^{34,36}$.

Ainda que 56,4\% dos entrevistados relatem conhecer a NR 32, esse número deve ser considerado insatisfatório, uma vez que a norma contém informações essenciais para as práticas diárias dos serviços de saúde. Conhecer e aplicar as normas dos trabalhadores da área da saúde é uma das competências específicas do estudante de Odontologia $^{20}$. Bragança et al. $(2010)^{33} \mathrm{em}$ pesquisa com cirurgiões-dentistas, observaram que $92,8 \%$ não conheciam a norma. Muitos profissionais já inseridos no mercado não tiveram acesso a essa informação durante sua graduação. Deve-se lembrar que a regulamentação da NR 32 é relativamente recente $^{37}$, assim como a intensificação da criação de condutas de biossegurança e a cobrança na execução delas, tanto por parte das instituições fiscalizadoras como por parte dos próprios funcionários e pela sociedade.

O conhecimento dos acadêmicos sobre como evitar $(93,3 \%)$ e proceder $(81,5 \%)$ em casos de acidentes com exposição a materiais biológicos demonstra a importância de que dos conteúdos de Biossegurança sejam abordados de maneira contínua durante a formação ${ }^{5}$. Entretanto, foi observado que os estudantes não realizaram as condutas pós exposição de maneira correta de acordo com o conhecimento recebido.

É importante lembrar que a NR 32 responsabiliza os empregadores quanto à capacitação de seus funcionários antes do início de suas respectivas atividades e que esta deve ser realizada de forma continuada $^{17,37,38}$. Os acadêmicos, nessa posição, são instruídos para atuar de maneira segura, mas assim que entrarem no mercado 
de trabalho devem ser capazes de realizar a gestão do processo das equipes de saúde em concordância com o conceito de saúde, bem como a seus possíveis funcionários ${ }^{20}$.

\section{CONCLUSÃO}

Os acadêmicos de Odontologia da amostra estudada se expuseram a acidentes com materiais biológicos, mesmo recebendo instruções de como evitá-los. Tais acidentes acometeram mais o sexo feminino, com maior incidência nas mãos, causados principalmente por sondas exploradoras e em Disciplinas que realizam, em sua maioria, procedimentos semicríticos. $\mathrm{O}$ uso correto do EPI foi negligenciado pela maioria dos acadêmicos acidentados. A conduta pós exposicional mais comum foi lavar o local com água e sabão e utilizar antisséptico. Houve subnotificação das ocorrências e pouca procura por atendimento médico após acidente com maior risco de contaminação. Os acidentes com exposição a materiais biológicos merecem maior atenção por parte dos docentes e das instituições de ensino, que devem desenvolver estratégias, incluindo supervisão mais eficaz e medidas contínuas que motivem e despertem a atenção dos acadêmicos para a redução do número dos acidentes e, ao mesmo tempo, valorizar sua notificação, sedimentando hábitos e condutas apropriadas para a vida profissional.

\section{ABSTRACT \\ Occupational accidents with biological material in Dentistry: a teaching responsibility \\ In Dental School, undergraduate students face situations that can cause health problems due to exposure to various occupational risks with biological material.}

In order to make contributions using data in academic training, the aim of this study is to verify the occurrence, characteristics of accidents with biological material, the knowledge and application of preventive actions, and post-exposure behavior. This observational, cross-sectional, and descriptive study was conducted using a self-administered questionnaire by 195 dental students who have started clinical procedures. Of the interviewees, $23.6 \%$ had occupational accidents with a higher frequency of females (78.3\%), commonly in the hands $(66.7 \%)$ and most of them caused by an explorer probe $(30.1 \%)$. It was observed that $34.9 \%$ had percutaneous accidents, but only $18.7 \%$ sought medical assistance and $6.3 \%$ reported not using Personal Protective Equipment (PPE) at the time of the exposure. Washing the affected area with soap and water $(54.7 \%)$ was the most common behavior performed postexposure. The occurrence of accidents with contaminated materials is alarming due to the incomplete use or non-use of PPE, in addition to the low demand for medical care after the injury. The students reported that they were aware of work health and safety legislation and how to proceed in case of occupational accidents. There is a need for the development of teaching strategies in a continuous way that include more effective supervision that motivates the students to reduce occupational accidents while increasing the number of students reporting incidents of biological exposure.

Descriptors: Exposure to Biological Agents. Occupational Risks. Accident Prevention. Students, Dental. Occupational Accidents Registry.

\section{REFERÊNCIAS}

1. Shaghaghian S, Pardis S, Mansoori Z. Knowledge, attitude and practice of dentists towards prophylaxis after exposure to blood and body fluids. Int $\mathbf{J}$ Occup Environ Med. 2014;5(3):146-54. 
2. Kon NM, Soltoski F, Júnior MR, Lozovey JCA. Acidentes de trabalho com material biológico em uma Unidade Sentinela: Casuística de 2.683 casos. Rev Bras Med do Trab. 2011;9(1):33-8.

3. Lima AA, Azevedo, AC, Fonseca AGL, Silva JLM, Padilha WWN. Acidentes Ocupacionais: Conhecimento, Atitudes e Experiências de Estudantes de Odontologia da Universidade Federal da Paraíba. Pesqui Bras Odontopediatria Clin Integr. 2008;8(3):327-32.

4. Oliveira MSSF, Porto AN, Semenoff TADV, Silva NF, Borges ÁH, Semenoff-Segundo A. Acidente com material biológico em graduando da área da Saúde. COORTE - Rev Científica do Hosp St Rosa. 2015; (5):31-6.

5. Pinelli C, Garcia PPNS, Campos JÁDB, Dotta EAV, Rabello AP. Biossegurança e odontologia: Crenças e atitudes de graduandos sobre o controle da infecção cruzada. Saúde Soc. 2011;20(2):448-61.

6. Pinelli C, Neri SN, Loffredo LCM. Dental students' reports of occupational exposures to potentially infectious biological material in a Brazilian School of Dentistry. Cad Saúde Coletiva. 2016;24(2):162-9.

7. Garbin AJI, Wakayama B, Garbin CAS. Negligência no autocuidado em saúde: a imunização contra a hepatite $\mathrm{B}$ na Odontologia. Arch Health Invest. 2016;5(2):85-9.

8. Ministério da Saúde. Secretaria de Atenção à Saúde. Exposição a Materiais Biológicos. Brasil; 2006 p. 76.

9. Ministério da Saúde. Boletim Epidemiológico - Hepatites Virais. Ano $\mathrm{V}, \mathrm{n}^{\mathrm{o}}$ 01, Revista Brasileira de
Medicina. Brasília - DF; 2016.

10. Shaghaghian S, Golkari A, Pardis S, Rezayi A. Occupational Exposure of Shiraz Dental Students to Patients' Blood and Body Fluid. J Dent (Shiraz, Iran). 2015;16(3):206-13.

11. Miotto MHMB, Rocha RM. Acidente ocupacional por material perfurocortante entre acadêmicos de Odontologia. Rev Bras Promoç Saúde. 2012;25(1):97-102.

12. Machado-Carvalhais HP, Martins TCP, Ramos-Jorge ML, Magela-Machado D, Paiva SM, Pordeus IA. Management of Occupational Bloodborne Exposure in a Dental Teaching Environment. J Dent Educ. 2007;71(10):1348-55.

13. Brasil. Ministério da Saúde (MS). Portaria ${ }^{0} 104$, de 28 de abril de 2004. 2004 p. 1-2.

14. Brasil. Ministério da Saúde (MS). Portaria $\mathrm{n}^{\mathrm{o}} 104$, de 25 de janeiro de 2011. 2011 p. 1-5.

15. Ministério da Saúde. Secretaria de Atenção a Saúde, Departamento de Ações Programáticas Estratégicas. Exposição a Materiais Biológicos. Brasil: Editora do Ministério da Saúde; 2006. 74 p.

16. Ribeiro $\mathrm{P}$, Hayashida $\mathrm{M}$, Moriya $\mathrm{T}$. Acidentes com material biológico entre estudantes de graduação em odontologia. Rev Odontol da Univ Cid S Paulo. 2007;19(3):263-8.

17. Garcia LP, Blank VLG. Condutas pósexposição ocupacional a material biológico na odontologia. Rev Saude Pública. 2008;42(2):279-86.

18. Lima AVM, Sousa LV, Carlos MX, Martins MGA, Pereira CKK, Pereira LSL. Prevalência e fatores de risco de acidentes com materiais pérfuro- 
cortantes em alunos de graduação em odontologia. Braz J Periodontol. 2016;26(4):15-23.

19. Garbin AJI. Imunização contra a hepatite b e os acidentes ocupacionais: importância do conhecimento na odontologia. Rev Saúde Pesq. 2016;9(2):343-8.

20. Conselho Nacional de Educação Câmara de Educação Superior. Resolução CNE/CES 3 de 19 de fevereiro de 2002. Resolução CNE/CES 3, de 19 de fevereiro de 2002 [Internet]. Institui Diretrizes Curriculares Nacionais do Curso de Graduação em Odontologia. Diario Oficial Uniao. 04 mar 2002. [Acesso em 22 set. 2008]. 2002. Disponível em http://portal.mec. gov.br/cne/arquivos/pdf/CES032002.pdf

21. Diretrizes Curriculares Nacionais do Curso de Graduação em Odontologia Minuta 2 para 2a consulta pública online da divisão de capítulos e seções: capítulo I - das diretrizes, capítulo II das competências gerais. [Acesso em 29 mai. 2017]. Disponível em http://www. abeno.org.br/arquivos/downloads/2Con sulta onlineDCNsOdontologia.pdf

22. Orestes-Cardoso SM, Farias ABL, Pereira MRM, Orestes-Cardoso AJ, Cunha IF. Acidentes perfurocortantes: prevalência e medidas profiláticas em alunos de odontologia. Rev Bras Saúde Ocup. 2009;34(119):6-14.

23. Kallás AR, Almeida CR. Acidentes ocupacionais com material biológico : a atuação do enfermeiro do trabalho. Enferm Rev. 2013;16(3):197-206.

24. Gir E, Netto, JC, Malaguti SE, Canini SRMS, Hayashida M, Machado AA. Acidente com material biológico e vacinação contra hepatite $B$ entre graduandos da área da saúde. Rev Latino-Am Enferm. 2008;16(3):401-6. 25. Artuzi FE, Bercini F, Azambuja TWF. Acidentes pérfuro-cortantes na Faculdade de Odontologia da Universidade Federal do Rio Grande do Sul. Rev Fac Odontol Porto Alegre. 2009;50(2):26-9.

26. Lima LKOL, Tipple AFV, Barros DX, Ferreira PS, Paiva EMM, Simões LLP. Acidentes com material biológico entre estudantes de odontologia no Estado de Goiás e o papel das instituições de ensino. ROBRAC. 2012;21(58):553-9.

27. Martins RJ, Garbin CAS, Garbin, AJÍ, Prieto AKC. Conhecimento e atitudes de profissionais da saúde frente à exposição ocupacional a material biológico. Cienc Trab. 2011;13(40) :113-5.

28. Sasamoto SAA, Tipple AFV, Leles CR, Silva ÉT, Paiva EMM, Lopes-Lima LKO. Perfil de acidentes com material biológico em uma instituição de ensino odontológico. Acta Sci - Heal Sci. 2014; 36(1):43-9.

29. Araújo TME, Silva NC. Acidentes perfurocortantes e medidas preventivas para hepatite $\mathrm{B}$ adotadas por profissionais de Enfermagem nos serviços de urgência e emergência de Teresina, Piauí Accidents. Rev Bras Saúde Ocup. 2014;39(130):175-83.

30. Khalil SDS, Khalil OAK, Lopes-Júnior LC, Cabral DB, Bomfim EO, Landucci LF, et al. Occupational exposure to bloodborne pathogens in a specialized care service in Brazil. Am $\mathbf{J}$ Infect Control. 2015;43(8):e39-41.

31. Martins AMEBL, Perreira RD, Ferreira RC. Adesão a protocolo pós- exposição ocupacional de acidentes entre 
cirurgiões dentistas Compliance with occupational post- exposure protocol for injuries among. Rev Saúde Pública. 2009;44(3):528-40.

32. Nogueira SA, Carvalho BKG, Medeiros AR, Carneiro SER, Souza GCA. Prevalence and work accident notification with exposure to biological material in Dentistry. Rev Ciência Plur. 2016;2(1):102-19

33. Bragança DPP, Fernandes MMSC, Júnior LF, Júnior ED. Condutas do cirurgião-dentista frente a acidentes biológicos. Odonto. 2010;18(35):24-9.

34. Kotze MJ, Labuschagne W. A method of determining the presence of blood in and on a dental needle after the administration of local anesthetic. J Am Dent Assoc. 2014;145(6):557-62.

35. Wood AJ, Nadershahi NA, Fredekind RE, Cuny EJ, Chambers DW. Student occupational exposure incidence: perception versus reality. J Dent Educ. 2006;70(10):1081-8.
36. Galdino A, Santana VS, Ferrite S. Os Centros de Referência em Saúde do Trabalhador e a notificação de acidentes de trabalho no Brasil. Cad Saude Pública. 2012;28(1):145-59.

37. Brasil. Ministério do Trabalho e Emprego. NR 32 - Segurança e saúde no trabalho em serviços de saúde. Portaria MTE n. ${ }^{\circ} 485$, de 11 de Novembro de 2005 (DOU de 16/11/05 - Seção 1) Brasil; 2005 p. 1-40.

38. Galon T, Marziale MHP, Souza WL. A legislação brasileira e as recomendações internacionais sobre a exposição ocupacional aos agentes biológicos. Rev Bras Enferm. 2011;64(1):160-7.

Correspondência para:

Seani Neumann de Paiva e-mail: sndpaiva@ gmail.com

Rua Maranhão, 285/1004 Praia da Costa 29101-340 Vila Velha, ES 